\title{
Quick Inspection of Power-plane Short Fault on Multichip Module
}

\author{
Fang-Lin Chao* and Ruey-Beei Wu \\ *Industrial Technology Research Institute, Hsinchu, Taiwan \\ Department of Electrical Engineering, National Taiwan University
}

Taipei, Taiwan, Republic of China

\section{ABSTRACT}

In this studies, current source is applied on two diagonal corners of the power plane. By changing the current source to another pair of the opposite corners, one can determine the associated zero voltage contour line. The intersection of the two zero voltage contour lines locates the position of the short fault. Analytical formula has been derived to give the potential distribution for the plane with rectangular shape. The excellent agreement with the exact short point validates the present inspection approach.

\section{INTRODUCTION}

Together with evolvement of integrated circuit technology is the ever increasing device density, switching speed, and system performance [1]. Multilayer structure has become the essential trend of substrate design to increase the interconnection density $[2,3]$. For high performance multichip module, the existence of numerous contact points representing greater challenge to fabricate and inspect the substrate. Since the inspection facility and the labor cost is increasingly expansive, inspection reduction is important for reducing manufacturing cost.

One of the common faults in multilayer board or MCM is the power plane short problem. The different power and ground planes might be shorted due to manufacturing variations, defects in via formation process, and so on. Automatic optical inspection (AOI) techniques had been widely utilized in printed circuit board inspection. The circuit pattern on laminated layer is scanned by an computer controlled optical beam and the resultant image is compared with the golden sample. The abnormal pattern located by AOI is then corrected by technician. However, AOI can not inspect all the short problems. For example, it can not detect the defects incurred by vias in the laminating process. Besides, AOI is mainly developed for structures with laminated substrates. The inspection of MCM-D substrate is difficult to fulfill by such an optical technique.

\section{INSPECTION OF POWER-PLANE SHORT FAULT}

Two inspection approaches of power plane short fault are investigated and compared in this study. One is to impose a dc voltage source between the power plane and the ground plane. As shown in Fig. 1, the current flows from the power plane, through the short, and to the ground plane. The short point can be located by probing voltage distribution on the substrate. One can measure the voltage values at power pads. The lower the value, the closer the pad is to the short point.

However, most MCM substrates have only a few positions convenient for voltage measurement. On occasion, these available probing points are not close to the short location. This make the inspection difficult and time consuming

The other approach is to drive the current through the power plane only. As shown in Fig. 2, current source is applied between two diagonal corners of the substrate. Due to the finite conductivity of the conductor, a potential distribution is established on the power plane. If there is a short point on the substrate, the potential of this point is equal to that of any point in the ground plane. Choosing the ground plane as the voltage reference, the fault will locate on the zero voltage contour line. By changing the current source to another pair of the opposite corners, one can determine the associated zero voltage contour line. The intersection of the two zero voltage contour lines locates the position of the short fault.

To be explained later, this approach requires few probing points to determine the zero voltage contour line. It works well even if the short point is not close to any of the probing points. Therefore, the approach is more convenient for MCM inspection.

\section{CALCULATION OF POTENTIAL DISTRIBUTION}

Consider the experimental setup shown in Fig. 2 where the plane is of size $a \times b$ and $\left(x_{s}, y_{s}\right)$ is the unknown short point. A current of amplitude $I$ flows from the driving point $\left(x_{1}, y_{1}\right)$, distributcs in the plane, and merges to the sink point $\left(x_{2}, y_{2}\right)$. 
Choosing the short point as the voltage reference, it can be shown that the voltage distribution $\mathrm{V}(\mathrm{x}, \mathrm{y})$ in the plane satisfies [4]

$$
-\left(\frac{\partial^{2} \mathrm{~V}}{\partial \mathrm{x}^{2}}+\frac{\partial^{2} \mathrm{~V}}{\partial \mathrm{y}^{2}}\right)=\frac{\mathrm{I}}{\sigma_{\mathfrak{a}}}\left[\delta\left(\mathrm{x}-\mathrm{x}_{1}\right) \delta\left(\mathrm{y}-\mathrm{y}_{1}\right)-\delta\left(\mathrm{x}-\mathrm{x}_{2}\right) \delta\left(\mathrm{y}-\mathrm{y}_{2}\right)\right]
$$

Subject to the homogeneous Neumann boundary condition. By applying the eigenfunction expansion method, the voltage distribution can be solved to be

$$
\mathrm{V}(\mathrm{x}, \mathrm{y})=\frac{\mathrm{I}}{\sigma_{\mathrm{o}}}\left[\tilde{\phi}(\mathrm{x}, \mathrm{y})-\tilde{\phi}\left(\mathrm{x}_{\mathrm{s}}, \mathrm{y}_{\mathrm{s}}\right)\right]
$$

$$
\begin{aligned}
& \tilde{\phi}(x, y)=\sum_{m, n=0}^{\infty} \frac{\epsilon_{m}, \epsilon_{n}}{a b} \frac{\cos \frac{m \pi x_{1}}{a} \cos \frac{n \pi y_{1}}{b}-\cos \frac{m \pi x_{2}}{a} \cos \frac{n \pi y_{2}}{b}}{\left(\frac{m \pi}{a}\right)^{2}+\left(\frac{n \pi}{b}\right)^{2}} \\
& \times \cos \frac{m \pi x}{a} \cos \frac{n \pi y}{b}
\end{aligned}
$$

and

$$
\epsilon_{\mathrm{m}}= \begin{cases}1 & \text { if } \mathrm{m}=0 \\ 2 & \text { if } \mathrm{m} \neq 0\end{cases}
$$

To facilitate the numerical computation, other representations which involve exponentially decaying series should be resorted to $[5]$.

For the first inspection approach where the current source is across the power and ground planes, the short point is just the current sink. Fig. 3 shows the potential for the case with $\mathrm{a}=45, \mathrm{~b}=50,\left(\mathrm{x}_{1}, \mathrm{y}_{1}\right)=(4.8,48)$ and $\left(\mathrm{x}_{2}, \mathrm{y}_{2}\right)=$ $(19.2,28.8)$. Owing to the higher current density at the short point, the potential gradient there is much greater then that in other region. From the analysis, we conclude that the short point locates in the maximum voltage gradient region.

Secondly, the inspection approach with current driving across two opposite corners in the power plane is analyzed. Fig. 4. shows the equi-potential contours for the case with $\left(x_{1}\right.$, $\left.\mathrm{y}_{1}\right)=(4.8,48)$ and $\left(\mathrm{x}_{2}, \mathrm{y}_{2}\right)=(42.5,3.8)$. The maximum gradient positions happen at the driving and sinking points in the power plane. The voltage gradient at the short point is much smaller than the source regions. Few voltage measurements ( at least two ) are sufficient to identify the equal potential contour where the short point locates.

\section{EXPERIMENT}

A test board is built to validate of the inspection approach. For simplicity of the measurement, the test board is enlarged to $45 \mathrm{~cm} \times 50 \mathrm{~cm}$ in size. A short via between power and ground plane is artificially made at the indicated point $(19.2,28.8)$. In the first setup, the current source is applied to the two diagonal corners at $(4.8,48)$ and $(42.5,3.8)$. The equi-potential contour plot of the normalized potential distribution is shown in Fig. 4. By moving the voltage meter across the board, the measured voltages $V(x, y)$ on the plane are listed in Table. $1(a)$. Given that $V(x, y)$ is linearly related to $\bar{\phi}(x, y)$ as depicted by (2), the linear regression can be employed to find the relation $\mathrm{V}(\mathrm{x}, \mathrm{y})=-0.2966+2.493$ $\tilde{\phi}(\mathrm{x}, \mathrm{y})$. Hence, the equi-potential line where the short fault locates is the one with $\tilde{\phi}\left(x_{s}, y_{s}\right)=0.1180$. We can plot the line in the equi-potential contour map shown in Fig. 5 .

In the second setup, the current source is applied to the other diagonal corners at $(4.8,2.2)$ and $(42.5,46.5)$. The equi-potential contour of the normalized potential distribution $\bar{\phi}(\mathrm{x}, \mathrm{y})$ for the case can be obtained and is shown by the dashed lines in Fig. 5. Table 1(b) listed the values of $\bar{\phi}(\mathbf{x}, \mathbf{y})$ and the measured $V(x, y)$ at same probing points. In this case, the best fit function is $V(x, y)=0.1125+2.552 \bar{\phi}(x, y)$ by linear regression and the short fault lies in the equi-potential lines with $\tilde{\phi}\left(\mathrm{x}_{\mathrm{s}}, \mathrm{y}_{\mathrm{s}}\right)=-0.0435$.

The equi-potential line where the short fault locates is also plotted as a dashed curve in Fig. 5. Finding the intersection of the two equi-potential lines obtained in the two setups yields the short point $\left(x_{s}, y_{s}\right)=(20.1,29.1)$. The excellent agreement with the exact short point validates the present inspection approach.

\section{CONCLUSIONS}

The power /ground planes short fault can happen by mask mis-alignment, manufacturing variation, or defects in via formation process. In this studies, current source is applied on two diagonal corners of the power plane. The equi-potential contour line where the short fault locates can be found by the voltage measurement at as small as two probing points. Applying the current source to another two corners can find another equi-potential contour and then determine the short point. Analytical formula has been derived to give the potential distribution for the plane with rectangular shape. A test board was built and the short fault was successfully located. It demonstrates that this inspection approach is quite helpful in MCM repairing works. 
Table 1.

The measured voltages ( unit: $\mathrm{mV}$ ) at the probing points in a rectangular plate of size $45 \mathrm{~cm} \times 50 \mathrm{~cm}$ and the current source is imposed across two diagonal corners at (a) $(4.8,48.0)$ and $(42.5,3.8),(b)(4.8,2.2)$ and $(42.5,46.5)$.

(a)

\begin{tabular}{lccc} 
(a) & & & \\
& & $\mathrm{V}(\mathrm{x}, \mathrm{y})$ & $\dot{\phi}(\mathrm{x}, \mathrm{y})$ \\
\hline 8.60 & 7.0 & -0.65 & -0.0863 \\
8.60 & 16.2 & -0.36 & 0.0161 \\
8.60 & 19.2 & -0.22 & 0.0657 \\
8.60 & 29.9 & 0.45 & 0.3054 \\
8.60 & 42.0 & 1.54 & 0.7251 \\
16.0 & 10.0 & -0.77 & -0.1460 \\
16.0 & 19.1 & -0.39 & -0.0161 \\
16.0 & 26.8 & 0.04 & 0.1342 \\
16.0 & 39.0 & 0.79 & 0.4090 \\
28.0 & 14.7 & -1.06 & -0.3015 \\
28.0 & 23.8 & -0.53 & -0.1082 \\
28.0 & 37.5 & 0.14 & 0.1361 \\
38.8 & 5.6 & -2.58 & -0.9426 \\
38.8 & 16.2 & -1.44 & -0.4704 \\
38.8 & 29.9 & -0.48 & -0.1026 \\
38.8 & 40.5 & -0.05 & 0.0516 \\
\hline
\end{tabular}

(b)

\begin{tabular}{cccc}
$\mathrm{x}$ & $\mathrm{y}$ & $\mathrm{V}(\mathrm{x}, \mathrm{y})$ & $\tilde{\phi}(\mathrm{x}, \mathrm{y})$ \\
\hline 8.60 & 7.0 & 2.14 & 0.7707 \\
8.60 & 19.2 & 0.90 & 0.3310 \\
8.60 & 29.9 & 0.21 & 0.0823 \\
8.60 & 42.0 & -0.23 & -0.0790 \\
16.0 & 10.0 & 1.26 & 0.4310 \\
16.0 & 19.1 & 0.66 & 0.2247 \\
16.0 & 26.8 & 0.19 & 0.0606 \\
16.0 & 39.0 & -0.34 & -0.1352 \\
28.0 & 14.7 & 0.45 & 0.1048 \\
28.0 & 23.8 & -0.01 & -0.0586 \\
28.0 & 37.5 & -0.78 & 0.3461 \\
38.8 & 5.6 & 0.43 & 0.0819 \\
38.8 & 16.2 & 0.12 & -0.0328 \\
38.8 & 29.9 & -0.70 & -0.3410 \\
38.8 & 40.5 & -1.73 & -0.7482
\end{tabular}

\section{REFERENCES}

[1] E. E. Davidson and G. A. Katopis, "Package electrical design," in Microelectronic Packaging Handbook, R. R. Tummala and E. J. Rymaszewski Ed., Ch. 3., New York: Van Nostrand Reinhold, 1989.

[2] A. J. Blodgett, Jr., "Microelectronic packaging," Scientific American, Vol. 249, pp. 86-96, July 1983.

[3] T. Dixon, "Multilayer ceramics: the key to high density interconnections," Electronic Packaging \& Production, pp. 76-82, Feb. 1983.

[4] R. B. Wu, "Resistance computations for multi-layer packaging structure by boundary element method," IEEE Trans. Comp., Hybrids, Manuf. Technol., Vol. CHMT-15, pp. 87-96, Jan. 1992.

[5] B. Friedman, Principles and Techniques of Applied Mathematics, 1956

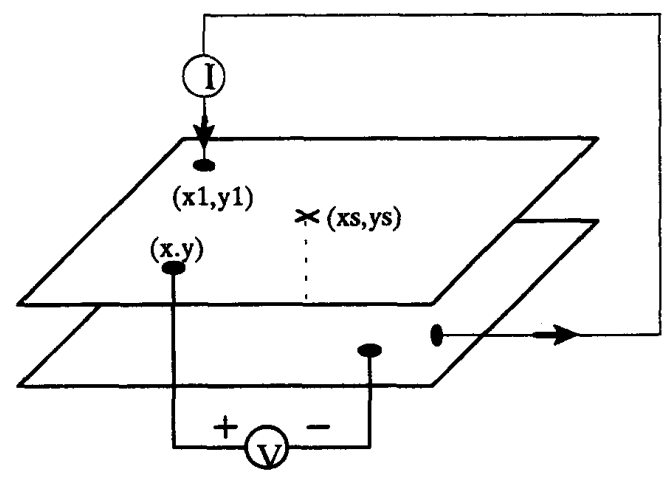

Fig.1 Inspection of power plane short fault by driving the current source across the power and ground planes. 


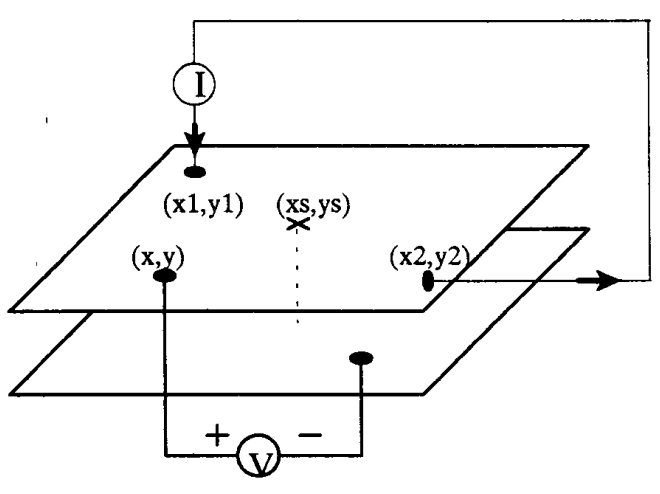

Fig.2 Inspection of power plane short fault by driving the current source across two diagonal corners at the power plane.

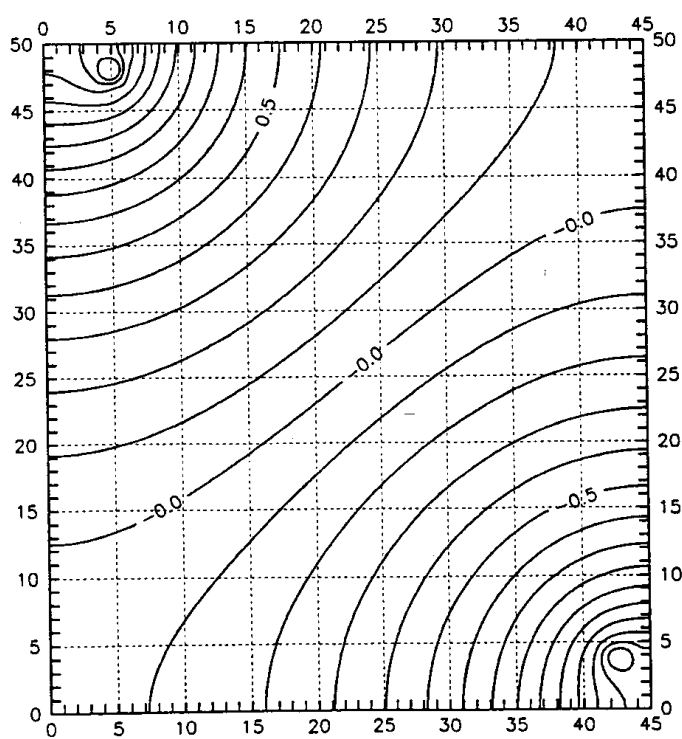

Fig.4 The equi-potential contour map in the power plane for the case with driving current source across two diagonal corners in the power plane.

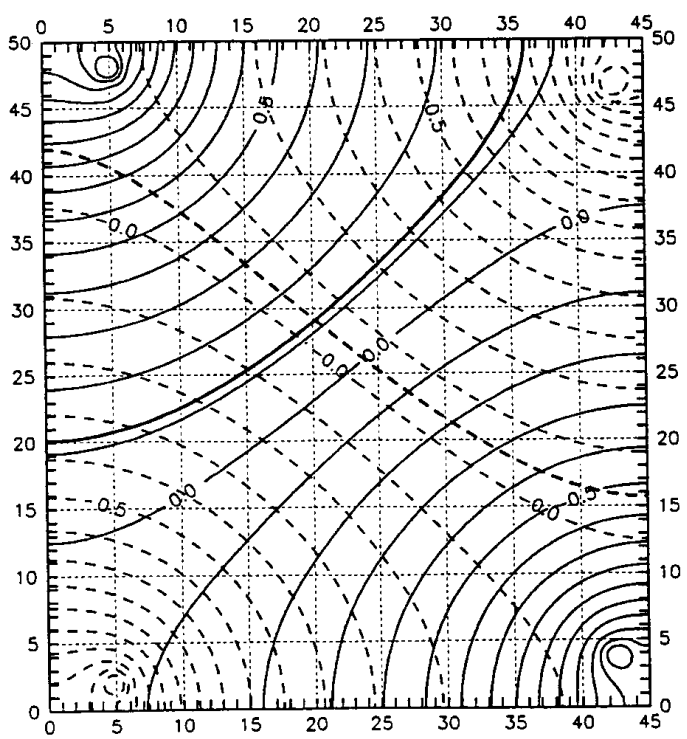

Fig.5 Determination of the short fault point from the intersection of where it locates the two equi-potential lines
Fig.3 The equi-potential contour map in the power plane for the case with driving current source across the power and ground planes.

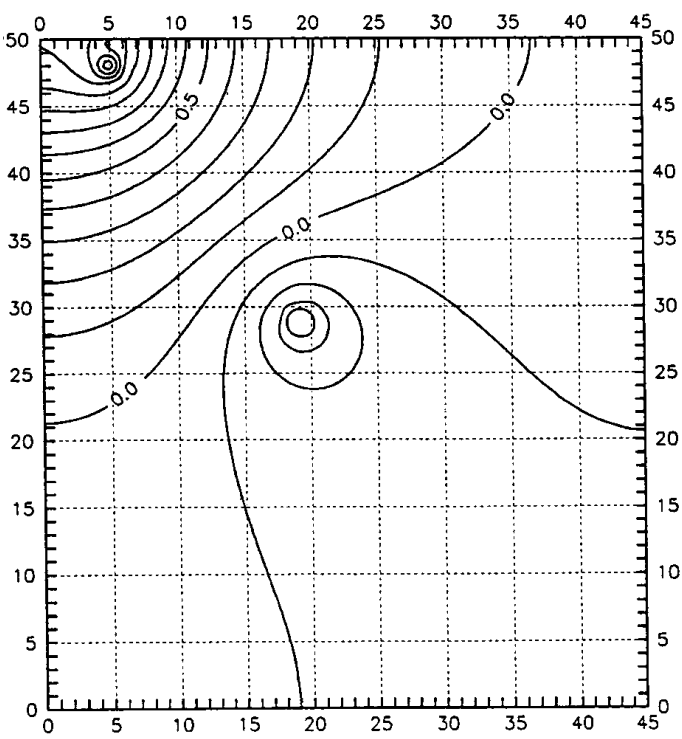

